\title{
When once is not enough: the case for repeated biopsychosocial assessments in psychosis
}

\author{
P. Dazzan*, Guest Editor and M. Tansella, Editor
}

First published online 27 May 2014

Although many clinicians still hold the Kraepelinian view of schizophrenia as an almost inevitably chronic and debilitating disease, many have moved to accepting schizophrenia (and psychoses in general) as a heterogeneous disorder with multiple possible courses and outcomes. This was also recently suggested by our 10-year follow-up of a large, epidemiological cohort of individuals first seen at the onset of any psychotic disorder (Morgan et al. 2014). In this cohort, we found that $46 \%$ of the subjects had remained free of psychotic symptoms for at least 2 years prior to follow-up, and this included $39.7 \%$ of those with a non-affective psychosis. Furthermore, we found that only $23 \%$ did not experience any remission during follow-up. If the course is so heterogeneous, why should we expect to understand the neurobiology of it by studying only one, or even two, predefined time points?

If we applied this approach to other conditions, such as for example recurring and remitting multiple sclerosis, would we know more about the neurobiology of this disorder? Of course, the answer is no, since the biology, as the symptoms, fluctuates in time. Still, we seem to have had the expectation that by studying psychoses at one time point, and by simply moving to evaluating earlier stages, we could understand more about what is really happening in the biological systems of individuals with these disorders. When we have tried to study multiple time points, we have simply studied predefined, fixed points in time, in isolation. One of the consequences has been the view that schizophrenia is a progressive disorder. Zipursky et al. (2013) recently published a provoking editorial in which they argue against this view. They support this position by discussing evidence that schizophrenia does not present with the progressive loss of function typical of deteriorating conditions, nor with cognitive loss, and also that some of the brain changes observed in longitudinal studies could be due to external factors. So, could schizophrenia be a fluctuating disorder, and

\footnotetext{
* Address for correspondence: Dr P. Dazzan, Department of Psychosis Studies, PO40, Institute of Psychiatry, King's College London, De Crespigny Park, London SE5 8AF, UK.

(Email: paola.dazzan@kcl.ac.uk)
}

if so, what are the factors that might affect what we see? In reality, we know very little about what happens in between evaluations.

The two editorials in this issue of EPS contribute to this debate providing two complementary and intriguing perspectives and some very important pointers for the direction to follow in research on the neurobiological basis of psychosis.

The Editorial by Cropley \& Pantelis (2014) discusses how, despite three decades of research, neuroimaging has actually contributed relatively little to our understanding of the pathophysiology of schizophrenia and is yet to have a clinical application. They propose that one of the main factors responsible for this has been the use of single assessments, which by providing a simple snapshot in time, ignore the importance of the trajectory of change. They start by discussing evidence from imaging studies on normal development and childhood-onset schizophrenia, which have provided evidence that brain regions develop in a non-linear fashion. As an example, they quote evidence that in childhood-onset schizophrenia, the normal parietofrontal pattern of progressive grey matter loss is more severe, but post-adolescence the grey matter loss slows down, becoming localised to prefrontal and temporal cortices. They then move to discussing studies of illness onset and the prodrome, which again suggest that these stages are characterised by active brain changes and again in a non-linear fashion. The authors argue that to understand these potential non-linear patterns of brain change, which could arise at different illness stages, and more importantly, in relation to concurrent symptoms, a re-conceptualisation of neuroimaging designs is required. Based on evidence that brain changes may fluctuate with changes in clinical state, they propose that frequent imaging assessments are needed to model the complex trajectories that occur with acute symptom changes. The authors propose that longitudinal designs in relation to changes in clinical status be employed to investigate neuroimaging markers of psychosis relapse. We shall also add that as well as being longitudinal in design, these neuroimaging studies should aim to evaluate large, epidemiologically representative samples of people suffering from 
psychosis. Most studies have been conducted, so far, on selected clinical samples, of individuals with an ongoing illness, thus by not including those most likely to be in remission from their illness. Importantly, Cropley \& Pantelis (2014) also point to a number of factors that could impact on the brain trajectories seen in acute schizophrenia, which among others include medications, substance use, lifestyle, genetics, stress and inflammatory processes. Relevant to the second Editorial in this Issue, they discuss how increases in brain volume seen with illness exacerbation could be interpreted to reflect brain 'swelling', which may be related to neuroinflammatory processes seen in the active stages of psychosis or relapse.

These neuroinflammatory and other factors are discussed more in detail in the second Editorial by Mondelli (2014). Here, she first discusses a body of evidence on the role of stress in precipitating the onset and relapse of psychosis, before moving on to discussing the possible biological mechanisms involved in the interaction between stress and psychosis onset. She discusses how a hyperactivation of the hypothalamic-pituitary-adrenal (HPA) axis, the main biological system involved in the stress response, seems to be part of the biological vulnerability to psychosis. Alterations in this axis have in fact been found not only at illness onset, but also prior to psychosis onset, and to even a greater extent among those individuals who subsequently develop frank psychosis. She points to the effect of HPA axis activation on neurogenesis and neuroplasticity as a possible mediator in the relationship between stress and psychosis. This is particularly important and relates to the previous editorial, since brain volume changes are evident at both the onset of psychosis or during the transition to psychosis, suggesting a critical role for neuroplasticity. The Editorial then discusses evidence that in both the onset of psychosis and the later stages of the disorder there is an increased inflammatory status. As for the HPA axis, inflammatory cytokines may also mediate the onset of psychosis by interaction with multiple pathways, including synaptic plasticity. This could in turn affect the brain structure, as suggested by evidence of an association between the increased inflammatory status, lower levels of neurotrophic factors and reduced hippocampal volume in patients at psychosis onset. Finally, the Editorial points to the complex interaction between these biological systems, antipsychotic medications and clinical stages. The point made throughout is that of an interplay between cortisol levels and symptom recovery, and of both a direct antiinflammatory and an indirect pro-inflammatory activity of antipsychotics, possibly mediated by their effect on weight-gain and increased adiposity. The Editorial concludes by suggesting that the stress biomarkers hold strong potential as predictors of psychosis as well as of clinical outcome, and may represent optimal targets for the development of novel therapeutic agents.

In summary, Cropley \& Pantelis (2014) suggest that changes may be related to the active illness stage and be temporary, and Mondelli (2014) points to biological systems that fluctuate, possibly in relation to external stimuli such as stressors. Considering that the brain may change along a non-linear trajectory, and in concomitance of a number of factors, should we then start paying more attention to what can affect the brain and ultimately the onset and course of psychosis? It is possible that some of the changes we see in the biological systems involved in psychosis are the indirect consequence of the illness, rather than part of its pathophysiology. That the adult brain is a highly plastic structure that responds to the environment is confirmed not only by neurological studies but also by studies in healthy individuals. A classical example is represented by the study of the effects of physical exercise on volume of the mid-temporal area and hippocampus in healthy individuals, even within relatively short periods of time (Draganski et al. 2004). If we can accept this evidence, then why not consider that some of the changes we see in the brain of individuals with psychosis are not only acute, but also perhaps due to the living conditions that can derive from having a serious disorder? Can living in an environment that is socially, physically and cognitively deprived negatively affect the plastic brain, and contribute to the changes we see in those individuals with a particularly poor outcome? One could consider the findings of our 6-year follow-up of firstepisode psychosis patients, where we found that while hippocampal volume had decreased in some patients, in others the volume had increased or remained the same (Lappin et al. 2013). Interestingly, these were the individuals who had better clinical, cognitive and functional outcomes. It can be tempting to say that a more aggressive illness is responsible for more marked (negative) brain changes, but we should go further and investigate whether this relationship is actually not a direct one, but one mediated by the environment in which the serious illness forces the affected individual to live.

We have much to learn from animal studies on the striking effects of enriched (or deprived) environments on plasticity processes of the adult brain (Sale et al. 2014). These can obviously be circular questions difficult to answer in humans, but they can be used to start thinking about changing the way we study psychosis. From our current approaches, we should move not only towards studying multiple time points in samples large enough to allow separate analyses of subgroups with different outcomes, but also to studying points that have a specific clinical meaning as well as environmental relevance, in terms of personal experience and living conditions. 
A history of childhood trauma, stress, substance misuse, medications, physical health and lifestyle can all interact with a variety of biological systems to ultimately shape psychosis course and outcome. These factors should be assessed in both social and biological studies of psychosis, with standardised measures that would not only improve the reliability of our findings, but also increase their comparability.

\section{Financial Support}

Paola Dazzan's research is supported by a NARSAD Independent Investigator Award and partly by the National Institute for Health Research (NIHR) Mental Health Biomedical Research Centre at South London and Maudsley NHS Foundation Trust and King's College London.

\section{Conflict of Interest}

None.

\section{References}

Cropley V, Pantelis C (2014). Using longitudinal imaging to map the 'relapse signature' of schizophrenia and other psychoses. Epidemiology and Psychiatric Sciences 23, 219-225.

Draganski B, Gaser C, Busch V, Schuierer G, Bogdahn U, May A (2004). Neuroplasticity: changes in grey matter induced by training. Nature 427, 311-312.

Lappin JM, Morgan C, Chalavi S, Morgan KD, Reinders AA, Fearon P, Heslin M, Zanelli J, Jones PB, Murray RM, Dazzan P (2014). Bilateral hippocampal increase following first-episode psychosis is associated with good clinical, functional and cognitive outcomes. Psychological Medicine 44, 1279-1291.

Mondelli V (2014). From stress to psychosis: whom, how, when and why? Epidemiology and Psychiatric Sciences (in press).

Morgan C, Lappin J, Heslin M, Donoghue K, Lomas B, Reininghaus U, Onyejiaka A, Croudace T, Jones PB, Murray RM, Fearon P, Doody G, Dazzan P (2014) Reappraising the long-term course and outcome of psychotic disorders: the AESOP-10 study. Psychological Medicine. First published online: 26 February 2014, doi: 10.1017/S0033291714000282.

Sale A, Berardi N, Maffei L (2014). Environment and brain plasticity: towards an endogenous pharmacotherapy. Physiological Reviews 94, 189-234.

Zipursky RB, Reilly TJ, Murray RM (2013). The myth of schizophrenia as a progressive brain disease. Schizophrenia Bulletin 39, 1363-1372. 